CRÍTICA, Revista Hispanoamericana de Filosofia

Vol. XXVII, No. 79 (abril 1995): 55-65

\title{
ANÁLISIS Y ELUCIDACIÓN: UN MÓDICO HOMENAJE A SIMPSON*
}

Manuel Comesaña

Sociedad Argentina de Análisis Filosófico

Universidad Nacional de Mar del Plata

\section{I}

Hace más de veinte años Alberto Coffa leyó en la Sociedad Argentina de Análisis Filosófico un trabajo titulado "Dos concepciones de la elucidación filosófica", que posteriormente fue publicado, junto con la respuesta de Thomas M. Simpson, por la revista Crítica (vol. VII, no. 21, diciembre de 1975, pp. 43-82). La contribución de Simpson a esa polémica se llamó “Análisis y eliminación: una módica defensa de Quine", y a ese título alude el que llevan estas líneas. En lo que sigue reseñaré brevemente la controversia y haré algunos comentarios sobre ella.

\section{II}

Coffa distingue dos concepciones de la elucidación [explication], y atribuye una de ellas a Quine. Según esta concepción, los objetos de la elucidación, los explicanda, son expresiones oscuras que aparecen en ciertos contextos que,

* Este trabajo fue leído en la mesa de homenaje a Thomas M. Simpson que se realizó el 15 de agosto de 1994 en el XIII Simposio Internacional del Insituto de Investigaciones Filosóficas de la UNAM. 
considerados en su conjunto, son lo suficientemente claros y precisos como para resultar útiles. Elucidar tales expresiones es encontrar otras que preserven los usos útiles y asignen valores de verdad arbitrarios a todas las demás oraciones en las que figuren las expresiones elucidadas. Un caso paradigmático de elucidación lo constituyen las definiciones de "par ordenado" formuladas por Wiener y Kuratowski. El único requisito de adecuación que debe satisfacer una definición de "par ordenado" es hacer verdadero el siguiente contexto:

$$
\text { Si }\langle x, y\rangle=\langle z, w\rangle \text {, entonces } x=z \text { y } y=w \text {. }
$$

Las definiciones de Wiener y Kuratowski hacen verdadero este contexto y asignan valores de verdad a todos los demás contextos en los que aparece la expresión " $\langle x, y\rangle$ ". La versión más conocida es la de Kuratowski, que identifica $\langle x, y\rangle$ con la clase $\{\{x\},\{x, y\}\}$, cuyos miembros son: (a) la clase $\{x\}$, cuyo único miembro es $x$, y (b) la clase $\{x, y\}$, cuyos miembros son $x$ y $y$. Según Coffa, la elucidación así entendida no contribuye a resolver, ni a disolver, los problemas filosóficos planteados por el explicandum, sino solamente a ignorarlos.

De acuerdo con la otra concepción — defendida por Tarski y Kreisel, entre otros-, el proceso elucidatorio consta de tres etapas: (1) identificación del explicandum; (2) formulación de las condiciones que debe satisfacer un explicatum aceptable, condiciones que constituyen un análisis parcial del explicandum; (3) formulación del explicatum, un concepto definido de manera precisa que satisface las condiciones identificadas en el segundo paso. En su respuesta a Coffa, Simpson ejemplifica este proceso con la noción de conocimiento proposicional. En primer lugar,

identificamos el objeto de nuestra elucidación diciendo que se trata del concepto expresado en contextos como "Juan 
sabe que la Luna es redonda", pero no en contextos como "Juan sabe manejar" o "Esto sabe a limón" [...] El paso 2 consiste en establecer, por ejemplo, que un explicatum adecuado debe conservar la verdad de la siguiente implicación: "Si $X$ sabe que $p$, entonces $p$ es verdadera", o sea que el conocimiento proposicional implica verdad [...] El tercer paso es dar el explicatum, que podría ser, aproximadamente, el que examina Platón en el Teeteto: $X$ sabe que $p$ si y sólo si: (a) $X$ cree que $p$; (b) $X$ tiene buenas razones para creerlo; (c) $p$ es verdadera (pp. 70-71).

El segundo paso no es - sostiene Coffa - una propuesta o estipulación sino una afirmación verdadera o falsa: el objeto seleccionado en la primera etapa puede no satisfacer las condiciones enunciadas en la segunda. Una elucidación puede ser, entonces, correcta o errónea. Será errónea si atribuye al explicandum rasgos que no le corresponden, o si deja de atribuirle rasgos que le son esenciales. Las elucidaciones de este tipo pueden ser útiles para la solución de problemas filosóficos; así, por ejemplo, entendemos mejor lo que es la verdad después de la elucidación de Tarski.

\section{III}

Sin embargo, ninguna elucidación puede ser un análisis del explicandum, ya que en todas el explicandum es vago y el explicatum, en cambio, es preciso, de modo que no pueden ser idénticos. Simpson expresó esto de manera muy clara y concisa, por lo cual me parece mejor volver a citarlo que parafrasearlo:

el explicandum no es simplemente una expresión que no exhibe a la luz del día los componentes conceptuales que pone de manifiesto el explicatum [...] el problema del análisis filosófico es que el explicandum posee grados variables de vaguedad, y no hay modo de establecer los límites exactos de su aplicación correcta; y si bien los criterios de adecuación — cuando son verdaderos - sirven para explicitar el núcleo 
identificable de su significado intuitivo, queda un resto de penumbra que la elucidación debe completar [...] clarificar un concepto es construir un concepto nuevo, con el margen de arbitrariedad que ello implica, pues el explicatum debe permitir o excluir claramente su aplicación a casos en relación con los cuales el término original se mantiene en silencio (pp. 71-72).

Coffa ha sostenido que hay dos clases de elucidaciones: las elucidaciones "à la Quine", que consisten en reemplazar una expresión por otra, y las elucidaciones "à la TarskiKreisel", que consisten en clarificar una noción captando su "significado real" —así se expresa Tarski en varios lugares-; las primeras son solamente un reemplazo, mientras que las últimas constituyen un análisis y son por eso las únicas que tienen importancia filosófica. La respuesta de Simpson consiste básicamente en señalar que un explicatum preciso no puede ser un análisis de un explicandum vago, y que toda elucidación tiene que ser, por lo tanto, un reemplazo. Es verdad que podemos equivocarnos en el segundo paso de la elucidación, esto es, en la identificación del núcleo preciso del explicandum; en esto Coffa tiene razón, pero no contra Quine, sino contra ciertos pasajes de Carnap donde éste sugiere que los requisitos de adecuación formulados en ese segundo paso deben ser vistos como una definición parcial implícita del explicandum. Quine no sostiene que sea arbitrario el proceso elucidatorio en su conjunto; sólo dice que el explicatum difiere arbitrariamente del explicandum, lo cual es cierto.

Coffa parece creer que hay una diferencia de clase entre, por ejemplo, la definición de "par ordenado" y la definición de "verdad" propuesta por Tarski, siendo la primera un mero reemplazo y la segunda, en cambio, un análisis genuino. Pero se trata en realidad de una diferencia de grado. Todas las elucidaciones tienen una parte de análisis - el análisis parcial que se realiza en el paso 2-y una 
de reemplazo; el predominio de una de esas partes puede hacer que la elucidación en su conjunto impresione como análisis o como reemplazo. Dicho predominio depende, por su parte, del grado de vaguedad del explicandum: cuando del uso ordinario sólo es posible extraer una condición, como ocurre en el caso del par ordenado, no es difícil tener la impresión de estar ante un reemplazo; cuando se pueden extraer varias - como sucede, por ejemplo, en el caso del conocimiento proposicional-, es más probable que el proceso impresione como un análisis.

\section{IV}

Lo que, a mi juicio, está en juego en esta discusión no es sólo la naturaleza de las elucidaciones sino la naturaleza de la filosofía en general. Aunque no lo dicen en forma clara y explícita, y mediante alguna frase dicha al pasar hasta sugieren lo contrario, tanto Quine como Coffa parecen dar por sentado que la filosofía se reduce a elucidaciones; al menos no hacen referencia a ninguna otra actividad filosófica, y uno de los parágrafos que Quine dedica al tema se titula "El par ordenado como paradigma filosófico" (y no "como paradigma de elucidación filosófica"). Dice Quine:

De acuerdo con una influyente doctrina de Wittgenstein, la tarea de la filosofía no es resolver problemas sino disolverlos mostrando que allí no había realmente ningún problema. Esta doctrina tiene sus limitaciones, pero se ajusta adecuadamente a la elucidación. Pues cuando la elucidación proscribe un problema lo hace mostrando que en un sentido importante el problema es irreal, a saber, en el sentido de que sólo procede de usos innecesarios (Word and Object, The MIT Press, 1960, p. 260).

Se equivocan los seguidores de Wittgenstein que deploran las elucidaciones porque se apartan del uso ordinario: 
"es precisamente mostrando cómo evitar las partes problemáticas del uso ordinario como mostramos que los problemas son puramente verbales" (p. 261).

Pero, como observa Coffa (p. 47), cualquier expresión problemática puede ser arbitrariamente reemplazada por otra; la cuestión es si el reemplazo preserva todo lo que hay que preservar. ¿Basta con preservar los “contextos útiles" de Quine? Según Coffa, no; es necesario establecer los "significados reales" de Tarski. Dejando a un lado sus posibles diferencias ontológicas, creo que en esta discusión no es necesario hacer distinciones entre esas dos cosas; se trata en ambos casos de afirmaciones que están implícitas en el uso corriente del lenguaje - corriente dentro de toda una comunidad lingüística natural o dentro de una comunidad más restringida de especialistas - , y que el análisis hace explícitas. Quizá no haga falta admitir, para entender las cosas de este modo, que esas afirmaciones estén en la mente del hablante - aunque no estoy nada seguro de que no estén ahí-; como lo ha señalado Simpson a propósito de los significados, podrían no estarlo,

del mismo modo que las leyes de la mecánica no están en la mente del ciclista que maneja de acuerdo con ellas; así como la mecánica proporciona la teoría de lo que el ciclista hace, la elucidación - podría sostenerse - ofrece la teoría de la práctica verbal del hablante, extrayendo de ella los criterios de aplicación que no están en su mente pero gobiernan su conducta lingüística (p. 77).

Sea como fuere, no todos los problemas filosóficos pueden resolverse, ni disolverse, haciendo explícito lo que está implícito en el uso ordinario. Algunas preguntas están implícitamente respondidas en dicho uso y otras no. Por ejemplo, la pregunta acerca de si el conocimiento implica verdad está respondida afirmativamente, como lo muestra el hecho de que no digamos "Los antiguos sabian que la 
Tierra era plana"; pero la pregunta acerca de cuáles pruebas no concluyentes bastan para que una creencia sea un conocimiento no parece estar respondida en modo alguno, y por eso el "problema de la inducción" no se puede disolver. No se trata de un seudoproblema originado en el mal uso que algunos filósofos hacen de términos como "racional", "buenas razones", etc., ni de un problema susceptible de solución "analítica", es decir, de uno que puede resolverse con sólo analizar el significado de esos términos. Aunque fuera cierto que razonar inductivamente forma parte del significado de la palabra "racional", también seguiría siendo cierto que los razonamientos inductivos no conservan la verdad. La pregunta “¿Por qué son confiables ciertos razonamientos que, sin embargo, pueden llevarnos de premisas verdaderas a conclusiones falsas?" expresa un problema genuino, salvo que los únicos problemas sean - como dice Coffa que creía Wittgenstein- "los que cuentan con solución presente y automática” (p. 62).

\section{V}

Coffa ha sostenido que, al menos en algunos casos, la elucidación no debe ser un reemplazo sino un análisis. Si sólo hubiera reemplazos, no existiría "la posibilidad del desacuerdo filosófico fuera del terreno de las actitudes" (p. 60). Si bien en algunas ocasiones "el reemplazo conceptual es condición esencial para el progreso intelectual" (por ejemplo, en física, en el tránsito a la teoría de la relatividad), "el monadismo intelectual extremo, la postulación del discontinuismo en la historia de la ideas - patente en Wittgenstein y latente en otros- [...] parece un error" (p. 63).

$[\mathrm{H}]$ ay algunos problemas que persisten a lo largo de procesos explicativos, [...] hay preguntas que - bajo formas diversas - se conservan a lo largo de la historia, preguntas y problemas cuya formulación se refina sin sufrir alteración 
esencial y que - suerte y genio mediante - reciben, al cabo, respuesta adecuada (p. 62).

Lo que Coffa está defendiendo es la idea de que hay problemas filosóficos, la idea de que la filosofía tiene derecho a existir. Me parece que esta idea es bastante fácil de compartir, pero creo también que Coffa elige mal el lugar que asigna a la filosofía - aunque tal vez sólo se trate de que su terminología no es suficientemente cautelosa, como dice Simpson a propósito de otra cuestión. A lo largo de toda su exposición - y a pesar de que ésta comienza con la frase: "Una buena porción de la filosofía de la ciencia contemporánea se ofrece como una serie de procesos de elucidación" (p. 43; las cursivas son mías)—, Coffa parece dar por sentado, como ya dije, que la filosofía se reduce a elucidaciones; si a esto se le agrega su tesis de que las elucidaciones deben ser análisis y no reemplazos, lo que se obtiene es una concepción de la filosofía como análisis del lenguaje, aunque en una variante especial. En su versión más difundida, la tesis de que la filosofía debe consistir en un análisis del lenguaje significa lo siguiente: algunas preguntas están implícitamente respondidas en el lenguaje ordinario y otras no; en lo que concierne a las primeras, la tarea del filósofo consiste en hacer explícito lo implícito; en cuanto a las otras, son seudopreguntas cuya formulación emplea ciertas palabras claves en un sentido distinto del corriente y, que por lo tanto, no está bien formular. Si Coffa no estuviera dispuesto a aceptar esto último - y es bastante probable que no simpatizara con prohibiciones de este tipo-, se vería obligado a sostener que todas las preguntas filosóficas están implícitamente respondidas en el uso común del lenguaje. Dicho de otro modo: algunos sostienen que no hay problemas filosóficos, y que el análisis del lenguaje ordinario permite disolver seudoproblemas; Coffa parece sostener, en cambio, que hay problemas filosóficos 
y que dicho análisis permite resolverlos. Pero la idea de que todas las preguntas filosóficas están respondidas en el uso común es poco verosímil (salvo que se la haga verdadera, como en la otra variante que acabamos de mencionar, mediante un círculo, agregando que las preguntas no respondidas en dicho uso son en realidad seudopreguntas).

Tal vez los problemas filosóficos no se resuelvan nunca; tal vez sea infundado el optimismo que se manifiesta en el último pasaje de Coffa que citamos, y quizás haya que conformarse con que se refine la formulación de las preguntas sin que nunca reciban respuesta adecuada. Sea como fuere, todo parece indicar que no bastan las elucidaciones para el tratamiento de esos problemas. Más aún: la filosofía propiamente dicha comienza después de las elucidaciones, como lo ha señalado Simpson:

Nadie ha visto nunca un número: sólo sabemos que dos más dos son cuatro, que cinco es menor que ocho, etc.; no es de extrañar, entonces, que en una elucidación de la aritmética a la manera clásica nos conformemos con elegir -o inventar - representantes de los números naturales entre los que resulten válidas las relaciones correspondientes. El verdadero problema filosófico comienza en rigor en este punto, en lugar de terminarse, como parece sugerirlo Quine (p. 79).

Quine podría responder que la pregunta “¿Qué es un número?" procede sólo de usos lingüísticos innecesarios y que una elucidación como la de Frege permite evitar esos usos sin perder nada importante. Un platonista podría contestarle, a su vez, que los números son entidades abstractas de cierto tipo - distintas de las clases-, y que seguramente muchas de sus propiedades más importantes todavía no han sido descubiertas, y no lo serán nunca si nos quedamos sólo con el explicatum de Frege. Tal vez Quine tiene razón, pero admitir que la tiene no puede ser algo anterior a la discusión filosófica. Por otra parte, la concepción quineana 
de la elucidación parece aplicarse solamente a preguntas de la forma: "¿Qué es un $x$ ?", y no, por ejemplo, a una pregunta como la que expresa el mencionado problema de la inducción. Tal vez no sea así, pero también esto debería ser el tema de una discusión filosófica. Alguien podría decir que esas aparentes discusiones son la escalera que se tira después de haber subido, pero hasta ahora la filosofía consiste solamente en escaleras, y no se sabe de nadie que ya esté arriba.

\section{VI}

No puedo poner fin a este "módico homenaje" sin decir algo sobre el libro que lo motiva y sobre su autor. Hace unos cuantos años, siendo estudiante de filosofía, leí Formas lógicas..., y quedé deslumbrado. El libro tenía todas las virtudes: originalidad, rigor, claridad, ingenio, y la mejor prosa filosófica que yo haya leído. Después me enteré de que era el primer libro de orientación analítica publicado originalmente en castellano, y el único en nuestro idioma que, en opinión de Alonzo Church, mostraba "una competente familiaridad con la bibliografía usual en este campo, y la capacidad para formular críticas significativas".

Dicen que conocer personalmente al autor de un libro que nos gustó mucho suele resultar decepcionante. Con Thomas me ocurrió todo lo contrario. Su inteligencia filosófica me impresionó al escucharlo, tanto como me había impresionado al leerlo; pero, además, nos hicimos amigos, y pude descubrirle otras virtudes: su bondad y su preocupación por el prójimo - a las que personalmente debo mucho-, y su honestidad intelectual, virtudes que lo llevan a prestarle a todos la misma atención sin reparar en grados de celebridad, y que en las discusiones lo hacen capaz de reconocer que el otro tenía razón sin sentir ninguna molestia. También llegué a conocer las circunstancias en 
que había sido escrito Formas lógicas. Es obvio que dichas circunstancias no pueden agregarle méritos al libro - cosa que, por otra parte, no necesita en lo más mínimo-, pero su conocimiento permite apreciar mejor los del autor. Al leer Formas lógicas, se tiene inevitablemente la impresión de estar frente a una obra madura, producto de muchos años de dedicación exclusiva al estudio de los difíciles problemas discutidos en el libro. Sin embargo, fue escrito en unos meses, mientras su autor, de poco más de treinta años, trabajaba como corrector de pruebas en una editorial.

También se dice que el prestigio de aquellos que conocemos personalmente tiende a parecernos inmerecido; con otras palabras, que nadie es famoso para sus amigos. Tampoco esto me sucedió: tengo la suerte de que Thomas sea mi amigo, pero para mí sigue siendo famoso.

Recibido: 19 de enero de 1995 